\title{
Harms of overoxygenation in patients with exacerbation of chronic obstructive pulmonary disease
}

\author{
Graeme Rocker MHSc DM
}

Cite as: CMAJ 2017 June 5;189:E762-3. doi: 10.1503/cmaj.170196

A nnual costs related to admissions to hospital for acute exacerbation of chronic obstructive pulmonary disease (COPD) were reported in 2007 at $\$ 750$ million. ${ }^{1}$ Acute exacerbation of COPD is still the primary reason for an adult hospital admission in Canada. A decade later, new data from the Ottawa Hospital, which spent about $\$ 20$ million on firsttime admissions for COPD $(n=1894)$ over four years, has shed more light on the reasons for this cost burden. ${ }^{2}$ The highest costs were for patients with COPD who were admitted to the intensive care unit (ICU), with a substantial association between costs and elevated partial pressure of carbon dioxide in arterial blood $\left(\mathrm{PaCO}_{2}\right)$. Uncompensated elevated $\mathrm{PaCO}_{2}$ causes respiratory acidosis, and acidemia can be deadly. ${ }^{3}$ It may seem intuitively right to put a "blue" patient on a high concentration of inspired oxygen $\left(\mathrm{FiO}_{2}\right)$. However, high $\mathrm{FiO}_{2}$ has been shown to be dangerous for patients with COPD, and guidelines advise against its use. Yet this dangerous practice continues, putting patients at risk of loss of life and using substantial health system resources.

Dangers of using high $\mathrm{FiO}_{2}$ for patients with COPD were welldocumented 60 to 70 years ago. ${ }^{4}$ Two more recent audits of COPD admissions in the United Kingdom that involved 983 and 9716 patients, respectively, ${ }^{3,5}$ reported associations between acidosis and the use of high $\mathrm{FiO}_{2}$. Moreover, the need for ventilatory support (in one in five patients) and high in-hospital mortality $(11 \%)$ followed use of high-flow oxygen. ${ }^{3}$ For patients with COPD, insufficient respiratory effort and/or inadequate alveolar ventilation, in a setting of uncontrolled oxygen delivery (where the precise $\mathrm{FiO}_{2}$ is unknown) can result in dangerous levels of both oxygen and carbon dioxide.

Physiologic mechanisms linking a raised $\mathrm{FiO}_{2}$ to hypercapnia are described in Appendix 1, available at www.cmaj.ca/lookup/ suppl/doi:10.1503/cmaj.170196/-/DC1. For example, if a patient were to be placed on a $100 \%$ oxygen nonrebreather mask for an extended period in response to an episode of distress and hypoxemia, $\mathrm{PaCO}_{2}$ might reach more than $150 \mathrm{~mm} \mathrm{Hg}$, partial pressure of oxygen in arterial blood $\left(\mathrm{PaO}_{2}\right)$ might reach more than

\section{KEY POINTS}

- Too much oxygen can be dangerous for patients with chronic obstructive pulmonary disease (COPD) with (or at risk of) hypercapnia (partial pressure of carbon dioxide in arterial blood greater than $45 \mathrm{~mm} \mathrm{Hg}$ ).

- Despite existing guidelines and known risk, patients with hypercapnia are often overoxygenated.

- Nasal prongs deliver pure oxygen to the nose, and patient factors (dead space and alveolar ventilation) determine final concentration at the alveolar level, thus use of nasal prongs can be dangerous in the context of acute exacerbation of COPD.

- A "safe sats" strategy (oxygen saturation by pulse oximetry of $88 \%$ to $92 \%$ ) that involves oxygen alert cards for hypercapneic patients, provision of Venturi masks and entering at-risk patients into an emergency health services database may avoid many episodes of iatrogenic acute hypercapnia and reduce overall morbidity, mortality and costs related to respiratory acidosis.

$250 \mathrm{~mm} \mathrm{Hg}$, and severe acidosis will occur with $\mathrm{pH}$ near 7.0. If this situation is present, patients like this can die a preventable iatrogenic death. Nevertheless, potential sources of high concentrations of uncontrolled oxygen (e.g., the 100\% nonrebreather mask and nasal prongs) are commonly used during hospital transfers, and treatment within emergency departments and in hospital wards. Nasal prongs provide uncontrolled oxygen by delivering pure oxygen to the nose without controlling the concentration. Partial pressure of oxygen $\left(\mathrm{PAO}_{2}\right)$ in the alveoli in a patient will depend on how much air is inspired simultaneously with the oxygen supplied by nasal prongs.

Before the British Thoracic Society guideline for emergency use of oxygen in adults that was published in $2008,{ }^{6}$ professional society guidelines for acute exacerbation of COPD focused little attention on the delivery of oxygen, although a 2003 Canadian Thoracic Society guideline had recommended $\mathrm{PaO}_{2}$ of $60 \mathrm{~mm} \mathrm{Hg}$ during acute exacerbation of bronchitis. Building on the study of the prevalence 
of acidosis in the UK, ${ }^{5}$ the British Thoracic Society recommended a target oxygen saturation by pulse oximetry $\left(\mathrm{Spo}_{2}\right)$ of $88 \%$ to $92 \%$ for patients known to be or at risk of becoming hypercapneic. Subsequently, a randomized controlled trial in Australia (involving 405 patients with known or suspected acute exacerbation of COPD), in which ambulance crews were randomly assigned to provide targeted $\mathrm{SpO}_{2}$ of $88 \%-92 \%$ or usual care with uncontrolled oxygen, reported lower mortality (odds ratio $0.22,95 \%$ confidence interval 0.05-0.91) and near normal $\mathrm{pH}$ among patients with COPD who received targeted $\mathrm{SpO}_{2}{ }^{7}$

A recent retrospective chart review in Halifax found the prevalence of hypercapnia with overoxygenation to be one in five among 89 patients who were subsequently enrolled in the INSPIRED COPD Outreach Program, which rose to one in two among 29 patients transferred to the ICU or intermediate care settings for bilevel positive airway pressure. ${ }^{8}$ Currently, hypercapneic patients within the INSPIRED program are issued an oxygen alert card and registered in the Emergency Health Services "at-risk" database, which recommends that paramedics provide a target $\mathrm{SpO}_{2}$ of $88 \%$ to $92 \%$ using the patient's own Venturi mask (provided through the INSPIRED program). Venturi masks deliver controlled oxygen at known concentrations via valves that are colour coded for a specific percentage of oxygen (e.g., $24 \%$ or $28 \%$ ). A subsequent audit confirmed high compliance with this strategy within the emergency department at the Halifax Infirmary. ${ }^{8}$

For patients with acute exacerbation of COPD, most hospital journeys begin with transportation by emergency health services. Recently, we conducted telephone interviews with ambulance services in five Canadian provinces (New Brunswick, Prince Edward Island, Saskatchewan, Ontario and Alberta) and determined that, despite awareness of the need for caution with oxygen delivery, none of the services used Venturi masks or had any standards or guidelines for care of hypercapneic patients. Target saturations for four provincial services (New Brunswick, Prince Edward Island, Saskatchewan and Ontario) exceeded the ranges recommended above. Ontario has a COPD-related standard for emergency services within the Basic Life Support Patient Care Standards; ${ }^{9}$ however, it is not in alignment with advice from professional societies. The standard for oxygen therapy in general is a target of $92 \%$ to $96 \%$. Ontario's standard recommends the following for patients with COPD “... increase oxygen by increments of two litres per minute above starting level approximately every two to three minutes if the patient's status deteriorates or the patient indicates they feel worse; and ... be prepared to ventilate." Our emergency health services teams are not to blame. Evidence-based guidance and proper training for emergency health services teams are required. Other health care providers have expressed concerns about lack of training and equipment for safe delivery of oxygen. ${ }^{10}$ However, even when guidelines for safe use of oxygen have been introduced, uptake has been slow. ${ }^{10}$

The guideline published in 2004 by the National Institute for Clinical Excellence in the UK affirmed that the aim of oxygen ther- apy for patients with COPD is to maintain adequate oxygenation without precipitating respiratory acidosis or worsening hypercapnia. ${ }^{11}$ Target saturations of $88 \%$ to $92 \%$ for this patient group are recommended currently by the British Thoracic Society ${ }^{6}$ and the 2017 Global Initiative for Chronic Obstructive Lung Disease report for treatment of hypoxia in acute exacerbation of COPD. ${ }^{12}$

The faithful practice of "safe sats" would likely avoid many episodes of iatrogenic acute hypercapnia and reduce overall morbidity, mortality and costs related to respiratory acidosis for patients with COPD. Placing patients with a known elevation of $\mathrm{PaCO}_{2}$ on a local ambulance service registry for target $\mathrm{SpO}_{2}$ of $88 \%$ to $92 \%$ using an inexpensive $(\$ 3.00)$ Venturi mask and following that advice on arrival in hospital could prove to be a simple and cost-effective strategy.

\section{References}

1. Mittmann N, Kuramoto L, Seung SJ, et al. The cost of moderate and severe COPD exacerbations to the Canadian healthcare system. Respir Med 2008;102:413-21.

2. Mulpuru S, McKay J, Ronksley PE, et al. Factors contributing to high-cost hospital care for patients with COPD. Int J Chron Obstruct Pulmon Dis 2017;12:989-95.

3. Roberts CM, Stone RA, Buckingham RJ, et al.; National Chronic Obstructive Pulmonary Disease Resources and Outcomes Project implementation group. Acidosis, non-invasive ventilation and mortality in hospitalised COPD exacerbations. Thorax 2011;66:43-8.

4. Westlake EK, Simpson T, Kaye M. Carbon dioxide narcosis in emphysema. $Q \mathrm{~J}$ Med 1955;24:155-73.

5. Plant PK, Owen JL, Elliott MW. One year period prevalence study of respiratory acidosis in acute exacerbations of COPD: implications for the provision of noninvasive ventilation and oxygen administration. Thorax 2000;55:550-4.

6. O'Driscoll BR, Howard LS, Davison AG; British Thoracic Society. BTS guideline for emergency oxygen use in adult patients. Thorax 2008;63(Suppl 6):vi1-68.

7. Austin MA, Wills KE, Blizzard L, et al. Effect of high flow oxygen on mortality in chronic obstructive pulmonary disease patients in prehospital setting: randomised controlled trial. BMJ 2010;341:c5462.

8. Rocker G, Kennedy H, Demmons J. Oxygen and hypercapnia: practicing "safe sats" [abstract]. Am J Respir Crit Care Med 2017;195:A5732.

9. Basic life support patient care standards. Toronto: Ontario Ministry of Health and Long-Term Care; 2016.

10. O'Driscoll BR, Bakerly ND, Caress AL, et al. A study of attitudes, beliefs and organisational barriers related to safe emergency oxygen therapy for patients with COPD (chronic obstructive pulmonary disease) in clinical practice and research. BMJ Open Respir Res 2016;3:e000102.

11. National Collaborating Centre for Chronic Conditions. Chronic obstructive pulmonary disease. National clinical guideline on management of chronic obstructive pulmonary disease in adults in primary and secondary care. Thorax 2004; 59(Suppl 1):1-232.

12. Global strategy for the diagnosis, management and prevention of chronic obstructive pulmonary disease 2017 report. Global Initiative For Chronic Obstructive Lung Disease; 2016.

Competing interests: Graeme Rocker has received honoraria from the Canadian Foundation for Healthcare Improvement.

This article was solicited and has not been peer reviewed.

Affiliations: INSPIRED COPD Outreach Program, Nova Scotia Health Authority; Department of Medicine, Dalhousie University; Division of Respirology, QEII Health Sciences Centre, Halifax, Nova Scotia; Canadian Foundation for Healthcare Improvement, Ottawa, Ont.

Correspondence to: Graeme Rocker, Gmrocker@dal.ca 